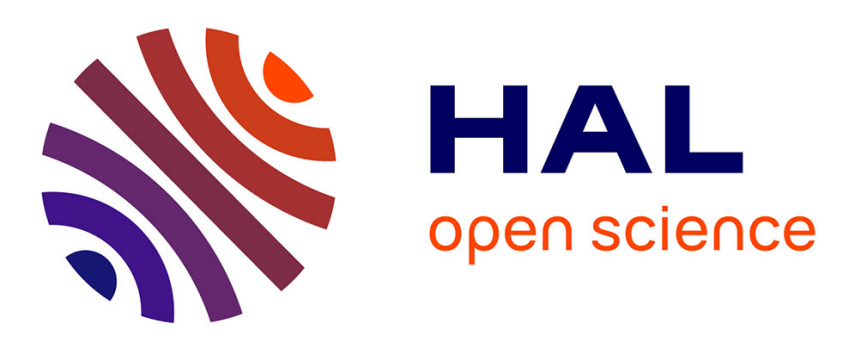

\title{
Non-linear spectral broadening across multiple bandgaps of all solid photonic crystal fibers
}

\author{
V. Pureur, J.M. Dudley
}

\section{To cite this version:}

V. Pureur, J.M. Dudley. Non-linear spectral broadening across multiple bandgaps of all solid photonic crystal fibers. SPIE photonics europe: Conference on Nonlinear Optics and Applications, Apr 2010, Bruxelles, Belgium. pp.77280D, 10.1117/12.854041 . hal-00563251

\section{HAL Id: hal-00563251 \\ https://hal.science/hal-00563251}

Submitted on 17 Apr 2021

HAL is a multi-disciplinary open access archive for the deposit and dissemination of scientific research documents, whether they are published or not. The documents may come from teaching and research institutions in France or abroad, or from public or private research centers.
L'archive ouverte pluridisciplinaire HAL, est destinée au dépôt et à la diffusion de documents scientifiques de niveau recherche, publiés ou non, émanant des établissements d'enseignement et de recherche français ou étrangers, des laboratoires publics ou privés. 


\title{
Non-linear spectral broadening across multiple bandgaps of all solid photonic crystal fibers
}

\author{
Vincent Pureur* and John M. Dudley \\ Institut FEMTO-ST, Département d'Optique P.M. Duffieux, CNRS UMR 6174, Université de \\ Franche-Comté, 25030 BESANCON, France.
}

\begin{abstract}
Nonlinear spectral broadening in two dimensional solid core photonic bandgap fibers is numerically investigated in the anomalous dispersion regime. A frequency-domain approach is used to simulate supercontinuum generation when femtosecond pulses are launched into a transmission band of a typical structure. The consequences on the output characteristics of the strong frequency dependence of the nonlinear parameter, the dispersion and the confinement losses of this kind of micro-structured fiber are highlighted, and we point out the necessity to include all of them in any numerical modeling of experiments. This numerical approach allows us to consider also the propagation of field energy in multiple photonic bandgaps simultaneously, and we show that efficient nonlinear spectral energy transfer is possible between adjacent and several photonic bandgaps across spectral regions of high attenuation.
\end{abstract}

\section{INTRODUCTION}

Two dimensional (2D) solid core (SC) photonic bandgap (PBG) fibers are one class of photonic crystal fibers (PCFs) in which light propagates within the solid core as a result of a PBG guidance mechanism ${ }^{1}$. These PCFs are generally constituted of a pure or doped silica core and high refractive index inclusions embedded in a pure silica host. As a result and in contrast to index guiding PCFs, the PBG guidance effect involves the propagation of the light across several distinct spectral transmission windows corresponding to PBG of different order, each associated with their own particular transmission characteristics, Group Velocity Dispersion (GVD) and nonlinearity. In particular, the PBG mechanism involves strong frequency-dependence of the waveguide losses (also called Confinement Losses (CL)), the effective-mode area (linked to the nonlinear parameter) and the GVD, almost close to each transmission band. As a consequence, 2D SC PBG fibers can combine the original optical properties of the PBG effect to a solid core, and are nowadays used as spectral filters for laser applications or nonlinear effects suppression ${ }^{2,3}$, as GVD compensator for solitonic propagation ${ }^{4}$ and for demonstration of nonlinear effects almost in the center of the transmission windows ${ }^{5}$. Whereas the original optical properties of each PBG in solid core structures would be expected to significantly influence nonlinear pulse propagation, only a few recent experimental studies were reported in this field until nowadays ${ }^{5-7}$, and no full and no systematic numerical studies have been performed to determine precisely how the different PBG characteristics impact on the spectral broadening in this kind of PCFs. We propose in this current work to investigate numerically, by a frequency-domain approach, femtosecond pulses supercontinuum generation in a typical solid core PBG structure, taking into account its strong frequency-dependent effective area, GVD and confinement losses. We show on the firsthand how these frequency-dependent properties impact on the supercontinuum output characteristics in the anomalous dispersion regime, and thus must all be included in any numerical modeling. On a secondhand, we simulate the propagation of field energy in the presence of multiple PBG order simultaneously. We show numerically that energy transfer is possible between different PBGs even in the presence of high attenuation spectral regions.

\section{FIBER STRUCTURE AND NUMERICAL METHOD}

In order to study spectral broadening in a two dimensional SC PBG fiber, we choose to simulate the optical properties of a hexagonal air-holes PCF infiltrated with high refractive index oil coming from the Cargille laboratories ( $\mathrm{n}_{\text {high }}=1.6$ at $589.3 \mathrm{~nm}, \mathrm{n}_{\text {low }}=\mathrm{n}_{\text {silica }}$ ). The low index core defect is constituted of one missing inclusion, and the fiber is supposed to be purely mono-mode in all the different PBGs. Structural inclusion diameter and pitch are $1.23 \mu \mathrm{m}$ and $3.1 \mu \mathrm{m}$ respectively ${ }^{8}$. Combined to a high refractive index contrast around $10 \%$ between the rods and the silica background, these opto-geometrical parameters lead to a first and second PBG order around $1.5 \mu \mathrm{m}$ and $800 \mathrm{~nm}$ respectively with a 
relatively large nonlinear parameter $\gamma$. Including the material dispersion of both the silica host and inclusion rods, the frequency-dependence of the group velocity dispersion, confinement losses (linked to the attenuation coefficient $\alpha$ ) and effective-mode area are computed with four confinement rings, and are shown in Fig. 1 for the four first PBG. As the different transmission windows of two dimensional solid core PBG fibers are separated by the 'cut-off' frequencies of low-order guided modes in one isolated high index inclusion (dashed vertical lines of Fig. 1), the optical properties of the selected fiber are plotted versus the normalized frequency $\mathrm{V}$ of a high refractive index rod.
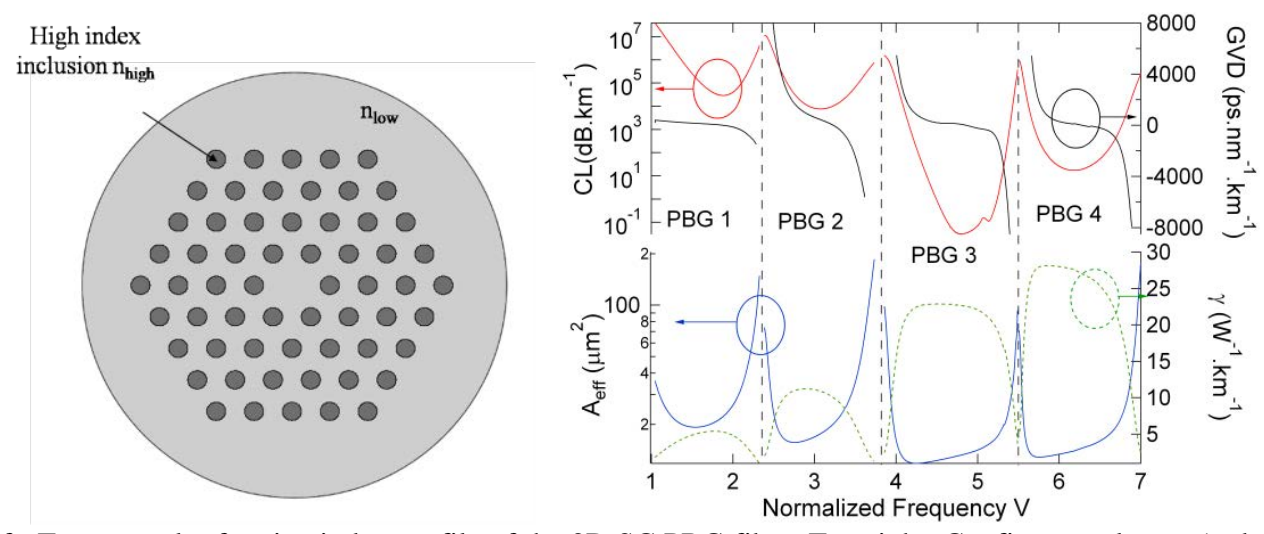

Figure 1. Left: Transversal refractive index profile of the 2D SC PBG fiber. Top right: Confinement losses (red curves, 4 confinement rings) and waveguide GVD (black curves) evolution within the four first PBG versus the normalized frequency $\mathrm{V}$ of an isolated inclusion. Bottom right: Effective area (blues curves) and nonlinear parameter (dashed curves) frequency evolutions for the PBG fiber. Dashed vertical lines are the low-order guided modes 'cut-off' in a high-index rod.

The frequency-dependent effective-mode area, called hereafter $\mathrm{A}_{\text {eff }}(\omega)$ (solid lines), and the associated nonlinear parameter $\gamma(\omega)$ (dotted lines) are represented on the bottom right of Fig. 1. Whereas the effective-mode area (nonlinear parameter) is a continuous increasing (decreasing) function of the wavelength in index-guiding PCFs, $\mathrm{A}_{\text {eff }}(\omega)$ is in the PBG structures a decreasing (rising) function of wavelength almost on the center of each PBG (this effect increasing with the PBG order). Figure 1 shows moreover several discontinuities close to each PBG edges where effective-mode area (nonlinearity) rises (fall) dramatically. Indeed, near each PBG edges, the fundamental guided-mode couple its energy from the core to cladding modes, affecting its effective-mode area, waveguide GVD and confinement losses which increase all to high values.

The strong frequency-dependence of all these parameters requires then a careful approach to their inclusion in any numerical scheme, and a convenient method is the frequency-domain implementation of the nonlinear propagation equation developed by Laegsgaard ${ }^{9}$. In this case, the generalized nonlinear Schrödinger equation for the pulse evolution takes the form:

$$
\frac{\partial \tilde{A}^{\prime}}{\partial z}=i \tilde{\gamma}(\omega) \exp (-i \tilde{L}(\omega) z) \mathfrak{I}\left\{\bar{A}(z, T) \int_{-\infty}^{\infty} R\left(T^{\prime}\right)\left|\bar{A}\left(z, T-T^{\prime}\right)\right|^{2} d T^{\prime}\right\}
$$

with the frequency dependent nonlinear parameter defined as:

$$
\bar{\gamma}(\omega)=\frac{n_{2} n_{0} \omega}{c n_{e f f}(\omega) A_{e f f}^{1 / 4}(\omega)}
$$

and

$$
\bar{A}(z, T)=\mathfrak{S}^{-1}\left\{\frac{\tilde{A}(z, \omega)}{A_{e f f}^{1 / 4}(\omega)}\right\}
$$


where $L(\omega)$ is the linear operator, given by $L(\omega)=\beta(\omega)-\beta\left(\omega_{0}\right)-\beta_{1}\left(\omega_{0}\right)\left[\omega-\omega_{0}\right]+i \alpha(\omega) / 2(\beta(\omega)$ being the propagation constant of the considered guided-mode related to $\mathrm{n}_{\mathrm{eff}}(\omega)$ by $\beta=\omega \cdot \mathrm{n}_{\mathrm{eff}} / \mathrm{c}$ and $\alpha(\omega)$ the attenuation coefficient), $\mathrm{R}(\mathrm{T})$ is the Raman response ${ }^{10}, n_{0}$ and $n_{2}$ the linear and nonlinear refractive index respectively of pure silica $\left(\mathrm{n}_{2}=2.6 \times 10^{-20} \mathrm{~m} / \mathrm{W}\right)$ and $c$ the speed of the light in the vacuum. This method is especially convenient as it allows for a direct implementation, with numerical solutions via standard suites of ordinary differential equation solvers, of strong frequency-dependent propagation constant $\beta$, attenuation coefficient $\alpha$ and effective mode area $A_{\text {eff. }}$.

\section{INFLUENCE OF THE FREQUENCY -DEPENDENT EFFECTIVE MODE AREA AND CONFINEMENT LOSSES}

We now present results using the numerical model described above to show how the different frequency-dependent parameters influence the nonlinear spectral broadening of femtosecond pulses supercontinuum generation. To this end, we firstly consider the conditions for which the spectral content is confined within only the PBG 1 shown on Fig. 1 with seven confinement rings and in the anomalous dispersion regime (zero dispersion wavelength at $1287 \mathrm{~nm}$ ). The frequency-dependent GVD is included in all cases and we consider the propagation of $100 \mathrm{~kW}$ peak power and $50 \mathrm{fs}$ (at FWHM) input pulses.

\subsection{Impacts on the supercontinuum output characteristics: highlighting}

Different output spectra are firstly computed for a propagation length of $50 \mathrm{~cm}$ within the PBG structure described in section 2 and for two pump wavelengths $\lambda_{\text {pump }}$ of $1.29 \mu \mathrm{m}$ and $1.55 \mu \mathrm{m}$. Figure 2 represents the intensity of the first ejected optical soliton for different cases: (i) full $\alpha(\omega)$ and full $\gamma(\omega)$ through all the considered PBG (red solid curves, realistic case); (ii) full $\alpha(\omega)$ but constant nonlinearity $\gamma\left(\omega_{0}\right)$ at the pump (blue dashed curves); (iii) $\alpha(\omega)=0$ but full $\gamma(\omega)$ (green dotted curves).

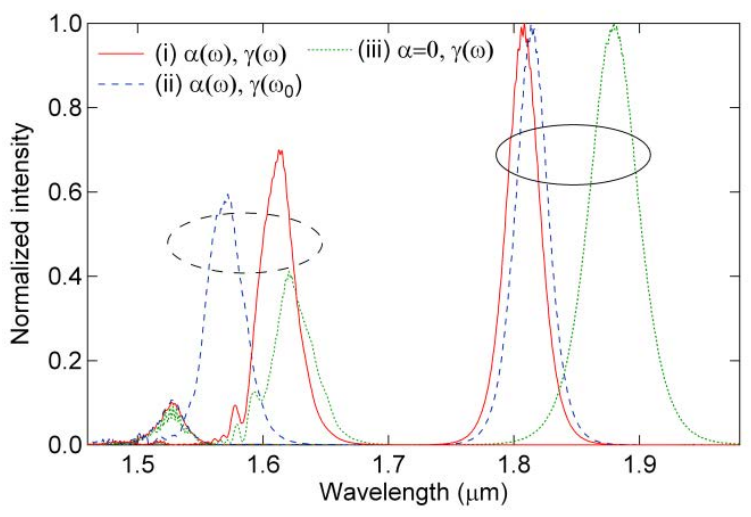

Figure 2. Output spectra (linear scale) simulated (i) with full $\alpha(\omega)$ and full $\gamma(\omega)$ (solid curves), (ii) full $\alpha(\omega)$ but constant $\gamma\left(\omega_{0}\right)$ (dashed curves), (iii) $\alpha=0$ but full $\gamma(\omega)$ (dotted curves). The pump wavelengths are $1.55 \mu \mathrm{m}$ (solid circle) and $1.29 \mu \mathrm{m}$ (dashed circle) (propagation distance of $50 \mathrm{~cm}$ ).

Figure 2 shows then significant differences between the realistic case (i) and cases (ii)-(iii). For a $1.29 \mu \mathrm{m}$ pump wavelength, the frequency-dependent effective-mode area involves on the generated optical soliton wavelength a spectral shift around $50 \mathrm{~nm}$ (case (ii)), whereas the shift induced by the confinement losses is weak. At the opposite, for a 1.55 $\mu \mathrm{m}$ pump wavelength, Fig. 2 highlights a spectral shift induced by the frequency-depend attenuation coefficient $\alpha(\omega)$ around $100 \mathrm{~nm}$ (case (iii)), whereas the shift induced by the nonlinearity becomes weak. These numerical results highlight for the first time that the strong frequency-dependence of both confinement losses and effective-mode area of solid core PBG structures can involve strong modifications on the first ejected optical soliton wavelength, and then on the supercontinuum output bandwidth. They suggest moreover that the spectral shift induced by both of them depend strongly on the pump wavelength. It is then clear that the strong frequency-dependence of nonlinearity, the GVD and the confinement losses of two dimensional solid core PBG fibers must be included in any numerical modeling to be suitable with experiments. 


\subsection{Effect of the propagation distance}

We previously observe that the spectral shift induced by the frequency-dependent nonlinearity $\gamma(\omega)$ and attenuation coefficient $\alpha(\omega)$ on the supercontinuum output bandwidth depend on the pump wavelength $\lambda_{\text {pump }}$ of the input pulse. This section shows that both $\gamma(\omega)$ and $\alpha(\omega)$ impacts on the first ejected optical soliton depend also strongly on the propagation distance. Figure 3 represents in the PBG1 the central optical soliton wavelength evolution versus fiber length for the three different cases previously described (pump wavelength of $1330 \mathrm{~nm}$ ), as well as for a fourth one, for which $\gamma=\gamma\left(\omega_{\circ}\right)$ through all the PBG and $\alpha=0$ (case (iv), black dashed curve).

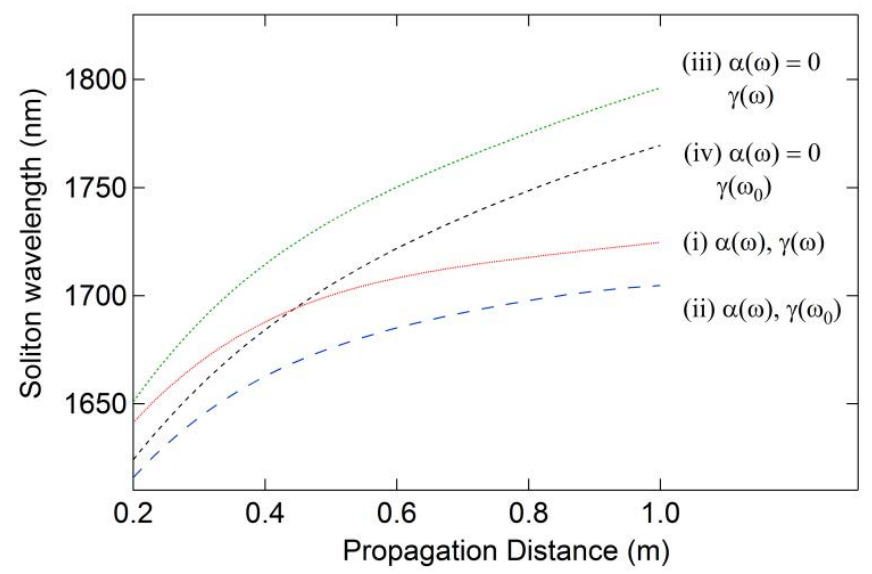

Figure 3. Soliton wavelength versus propagation distance for a $1330 \mathrm{~nm}$ pump with different frequency-dependence effects as described in the text.

Figure 3 shows that the frequency-dependent losses tend to reduce the supercontinuum output bandwidth. Indeed, confinement losses are very high close to the red edge of each PBG, and limit then the spectral red shifting of the optical soliton. The reduction of the supercontinuum bandwidth increases then with the accumulated losses, proportional to the propagation distance. Case (i) (green solid curve) of Figure 3 suggests finally that, for short propagation distance, the optical soliton wavelength (and so the supercontinuum bandwidth) is affected mainly by the frequency-dependent effective-mode area, whereas it is affected for long fiber length mainly by the confinement losses, which become important.

\section{ENERGY TRANSFER ACROSS MULTIPLE PHOTONIC BANDGAPS}

We focus now our investigations on a more challenging scenario, involving the propagation of field energy in multiples PBGs simultaneously. Previous work already point out experimental energy transfer from the $2^{\text {nd }} P B G$ to the $3^{\text {rd }} P B G$ when femtosecond pulses are launched into a hybrid photonic crystal fiber ${ }^{6}$. We firstly numerically investigate propagation of field energy into two adjacent PBGs of the structure described in section 2. Then we extend our analysis to the generation of nonlinear components into four different PBGs.

\subsection{Two adjacent Photonic Bandgaps}

We first simulate the propagation of $100 \mathrm{~kW}$ peak power and $50 \mathrm{fs}$ (FWHM) input pulses launched into the $2^{\text {nd }}$ PBG with a pump wavelength of $830 \mathrm{~nm}$ (zero dispersion wavelength at $790 \mathrm{~nm}$ ). We compute the spectral broadening occurring simultaneously into the $2^{\text {nd }}$ and $3^{\text {rd }}$ PBGs. The nonlinearity and losses are full frequency-dependent in both PBGs. Note that the different spectral transmission windows of 2D solid core PBG fibers are separated by high CL and effectivemode area regions, where no fundamental modes can be guided into the core. For these spectral regions, the constant propagation $\beta$ is set to zero whereas the $C L$ and effective-mode area are fixed to a constant maximal value. 

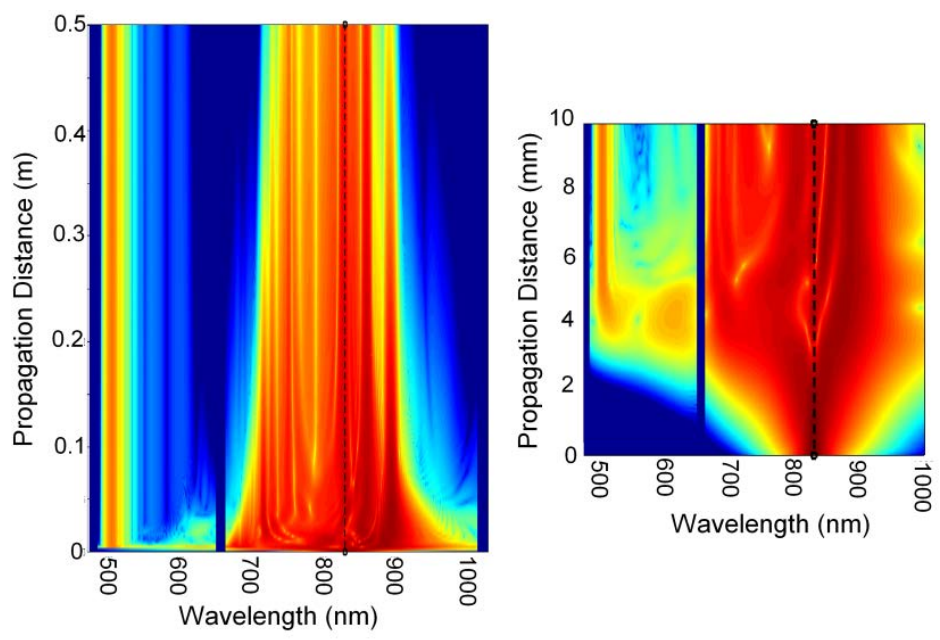

Figure 4. Density plots of the spectral field energy over fiber lengths of $50 \mathrm{~cm}$ (left) and $10 \mathrm{~mm}$ (right).

Figure 4 shows the computed spectral broadening as a function of the propagation distance into the $2^{\text {nd }}$ and $3^{\text {rd }}$ PBGs. We firstly observe, on the right of Fig. 4, the initial symmetric broadening of the input pulse, followed by the perturbation from higher order dispersion which results in the transfer of energy across the zero dispersion wavelength. The left of Fig. 4 shows clearly, on longer propagation distance, the fission of the input pulse to lower order optical soliton into the $2^{\text {nd }}$ PBG order. Figure 4 highlights moreover a consequent spectral broadening into the $3^{\text {rd }}$ PBG after a propagation length of $4 \mathrm{~mm}$.

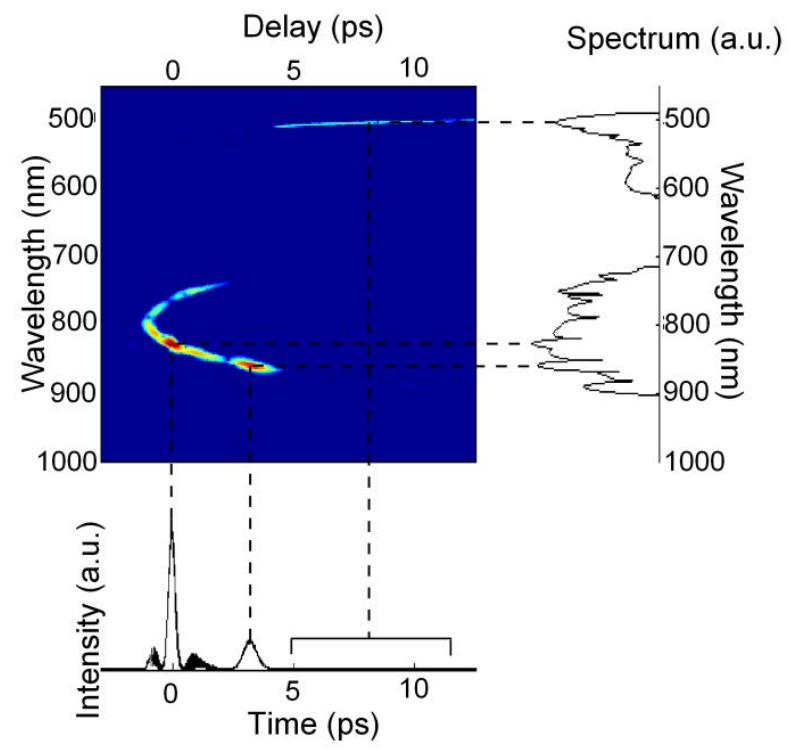

Figure 5. Simulated spectrogram projected into the temporal intensity and spectrum (propagation distance of $50 \mathrm{~cm}$ ).

Figure 5 represents a simultaneous representation of the temporal and spectral field (spectrogram) computed for a propagation distance of $50 \mathrm{~cm}$. This figure points out the generation, close to the blue edge (in wavelength) of the $3^{\text {rd }}$ PBG, of a dispersive wave phase-matched with the first ejected optical soliton into the $2^{\text {nd }}$ PBG order. Note that this numerical result can be surprising given the strong intermediate confinement losses between the $2^{\text {nd }}$ and $3^{\text {rd }}$ PBGs. It is then important to notice that the nonlinear length $\mathrm{L}_{\mathrm{NL}}$ is computed to be $\sim 0.8 \mathrm{~mm}$ only, and the losses integrated on this length are relatively low at $2.6 \mathrm{~dB}$. 
Note finally that these numerical results are in agreement with previous experimental work ${ }^{6}$, where authors relate the generation of a dispersive wave close to the blue edge into the $3^{\text {rd }}$ of a hybrid photonic crystal structure.

\subsection{Multiple Photonic Bandgaps}

We finally focus our investigations on the spectral broadening occurring across the four first PBGs of the structure described in section 2 and shown on the right of Fig. 1. The parameters are the same as previously mentioned, except for the input peak power which is set now to $200 \mathrm{~kW}$. Figure 6 shows the density plot of the spectral energy over a fiber length of $10 \mathrm{~mm}$ as well as the temporal field computed for a propagation distance of $40 \mathrm{~cm}$.
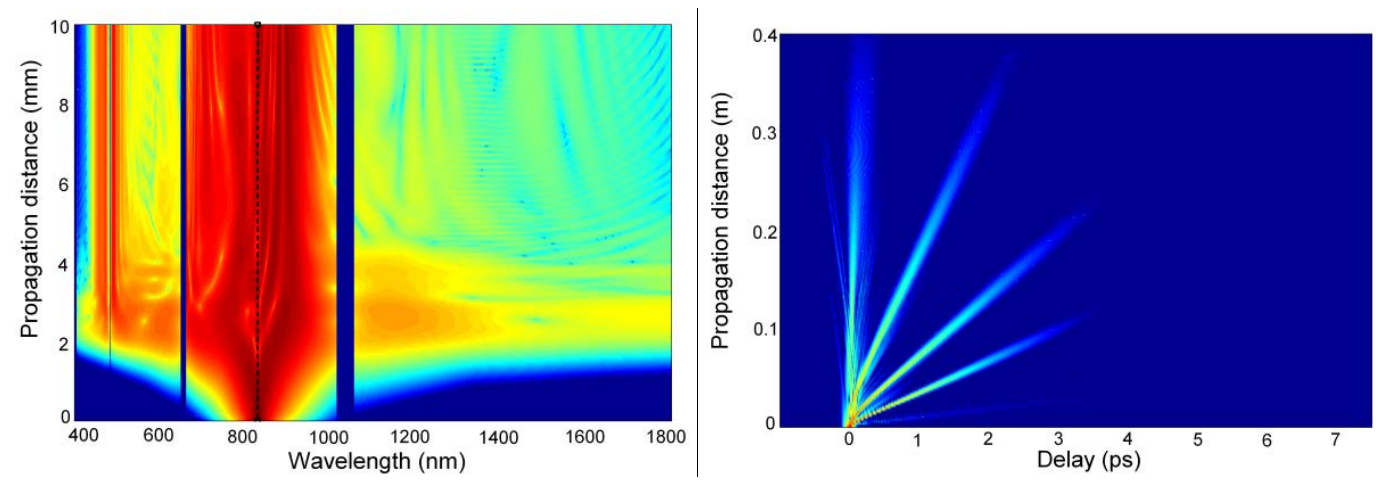

Figure 6. Left: Density plot of the spectral field energy over a fiber length of $10 \mathrm{~mm}$. Right: Temporal field evolution over a fiber length of $40 \mathrm{~cm}$. The launched peak power is set to $200 \mathrm{~kW}$ and the pump wavelength to $830 \mathrm{~nm}\left(2^{\text {nd }}\right.$ PBG).

As previously mentioned, the left of Fig. 6 shows the energy transfer from the $2^{\text {nd }} P B G$ to the $3^{\text {rd }} P B G$, but also to the $4^{\text {th }}$ PBG across a new inter-band with high attenuation around $470 \mathrm{~nm}$. Again, this energy transfer is possible because of the low intermediate confinement loss integrated on the weak nonlinear distance of $0.4 \mathrm{~mm}$ in this case. Figure 6 highlights for the first time energy transfer from the $2^{\text {nd }}$ PBG to a lower order PBG on the long wavelength side. This energy transfer seems however to be lower than the one occurring to the higher order PBGs because the confinement losses computed in the $1^{\text {st }}$ PBG with only 4 confinement rings are the highest $(33 \mathrm{~dB} / \mathrm{m}$ at the minimum). Note that confinement losses in 2D solid core PBG structures can be decreased for example by adding more rings in the microstructured cladding, and energy transfer to low order PBG can be improved.

\section{CONCLUSIONS}

We investigate numerically the propagation of femtosecond pulses into a two dimensional solid core photonic bandgap fiber. By a frequency-domain method, the spectral broadening occurring in such structures is computed taking into account the strong frequency-dependent nonlinearity, dispersion and confinement losses. We firstly show that all of them influence on the output supercontinuum characteristics like its bandwidth, and must be then included in any numerical model to predict accurately experimental measurements based on this class of PCFs. The frequency-domain approach allows moreover simulating simultaneously the propagation of field energy into two adjacent photonic bandgaps. We numerically highlight a possible energy transfer from the first ejected optical soliton to a phase-matched dispersive wave across high inter-band attenuation region. This energy transfer can occur because of the low confinement loss integrated over the characteristic nonlinear distance for initial spectral broadening. Our studies extend finally on the field energy propagation in multiple photonic bandgaps. We show possible energy transfer to several higher order photonic bandgaps compared to the pump, but also for the first time to a lower photonic bandgap order. 


\section{REFERENCES}

1. Pureur, V. et al., "Overview on Solid Core Photonic Bandgap Fibers," Fiber Integrated Optics 28, 27-50 (2009).

2. Pureur, V., Bigot, L., Bouwmans, G., Quiquempois, Y., Douay, M. and Jaouen, Y., "Ytterbium-doped solid core photonic bandgap fiber for laser operation around $980 \mathrm{~nm}$, " Applied Physics Letters 92, 061113 (2008).

3. Taru, T., Hou, J. and Knight, J. C., "Raman gain suppression in all-solid photonic bandgap fiber," $33^{\text {rd }}$ ECOC, Sept. 16-20, Berlin (2007).

4. Isomäki, A. and Okhotnikov, O. G., "Femtosecond soliton mode-locked laser based on Ytterbium-doped photonic bandgap fiber," Optics Express 14, 9238-9243 (2006).

5. Fuerbach, A., Steinvurzel, P., Bolger, J. and Eggleton, B., "Nonlinear pulse propagation at zero dispersion wavelength in anti-resonant photonic crystal fibers," Optics Express 13, 2977-2987 (2005).

6. Cerqueira, A. S., Cordeiro, C. M. B., Biancalana, F., Roberts, P. J., Hernandez-Figueroa, H. E. and Cruz, C. H. B., "Nonlinear interaction between two different photonic bandgaps of a hybrid photonic crystal fiber," Optics Letters 13, 2080-2082 (2008).

7. Kibler, B., Martynkien, T., Szpulak, M., Finot, C., Fatome, J., Wojcik, J., Urbanczyk, W. and Wabnitz, S., "Nonlinear femtosecond pulse propagation in an all-solid photonic bandgap fiber," Optics Express 17, 10393-10398 (2009).

8. Rasmussen, P. D., Laegsgaard, J. and Bang, O., "Degenerate four wave mixing in solid core photonic bandgap fibers," Optics Express 16, 4059-4068 (2008).

9. Laegsgaard, J., "Mode profile dispersion in the generalized nonlinear Schrödinger equation," Optics Express 15, 16110-16123 (2007).

10. Agrawal, G. P., [Nonlinear Fiber Optics], Academic Press, San Diego (2007).

11. Kibler, B., Dudley, J. M. and Coen, S., "Supercontinuum generation and nonlinear pulse propagation in photonic crystal fiber: influence of the frequency-dependent effective mode area," Applied Physics B: Lasers and Optics 81, 337-342 (2005). 